CONF-890718--29

DE 89017691

\title{
HIGH RESOLUTION-ANGLE RESOLVED PHOTOEMISSION STUDIES OF HIGH TEMPERATURE SUPERCONDUCTORS
}

C. G. Olson, R. Liu, D. W. Lynch

Ames Laboratory and Dept. of Physics, Towa State Univ., Ames, IA 50011

B. W. Veal, Y. C. Chang, P. Z. Jiang, J. Z. Liu, A. P. Paulikas

Materials Science Division, Argonne National Laboratory,* Argonne, IL 60439

A. J. Arko, R. S. List

Los Alamos Nátional Laboratory, Los Alamos, NM 87545

\section{AUGUST 1989}
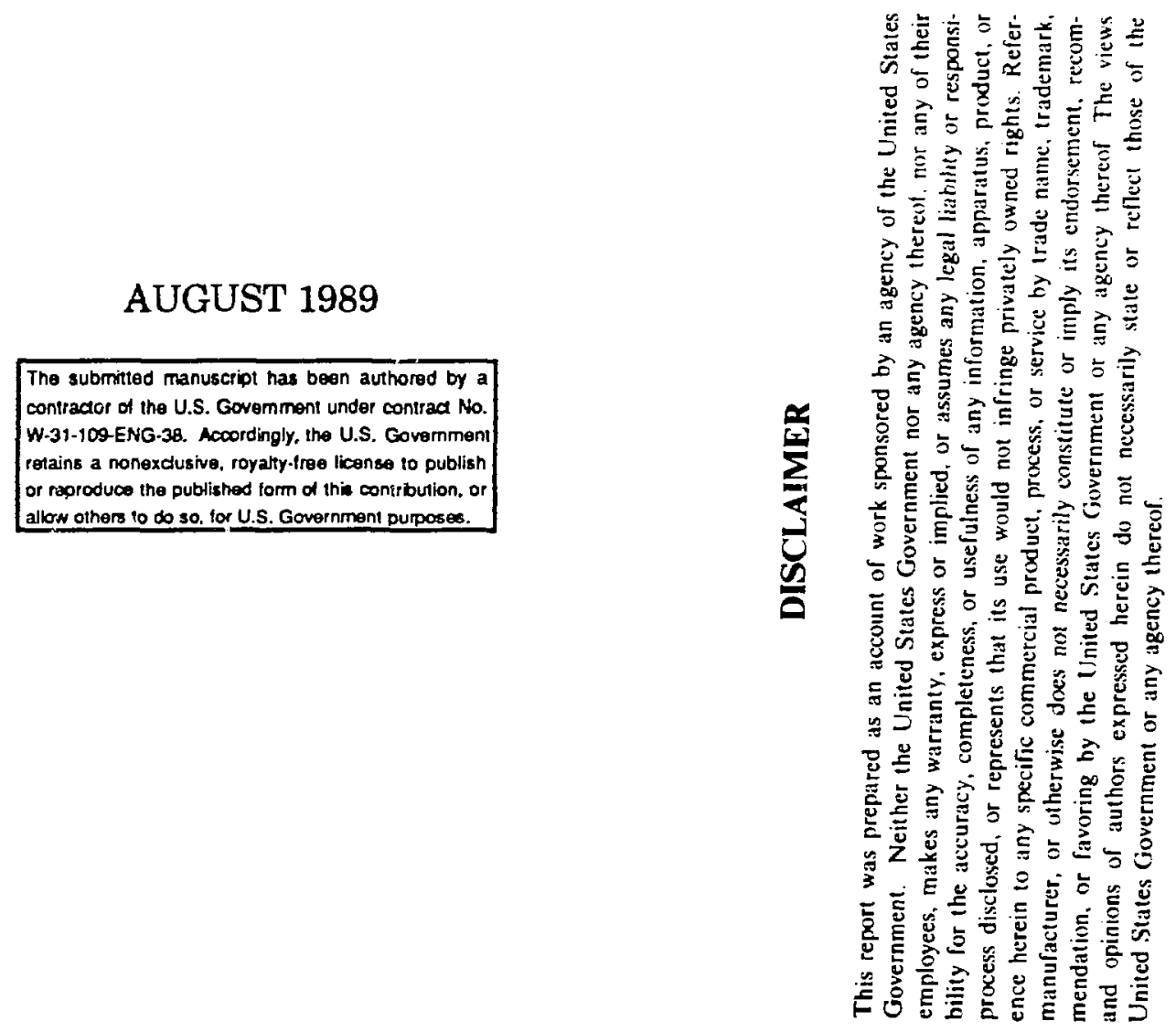

To be published in Physica C - Special Issue, Proc. of the Intl. M2S-HTSC Conference, Stanford, CA, July 23-28, 1989.

*Work supported by the U.S. Department of Energy, Basic Energy SciencesMaterials Science under Contract \#W-31-109-ENG-38. 
HIGH RESOLUTION-ANGLE RESOLVED PHOTOEMISSION STUDIES OF HIGH TEMPERATURE SUPERCONDUCTORS

C. G. OLSON", R. LIU, D. H. LYNCH

Ames Laboratory and Department of Physics, Iowe State University, Ames, IA 50011

B. H. VEal, Y. C. Chang, P. Z. JIANG, J. Z. LIU, A. P. PaUlikas

Argonne National Laboratory, Argonse, IL 60439

A. J. ARHO, R. S. LIST

Los Mlamos National Laboratory, Los Mlamos, NM 87545

Recent photoemission studies of Y 123 and B1 2212 performed with high energy and angular resolution have provided detalled information on the nature of the states near the Fermi level. Heasurements of the superconducting gap, band dispersion, and the density of states near the fermi level in the normal state all support a Fermi liquid description of these materlals.

\section{INIRODUCTION}

Historlcally, photoemission has had alxed success in determining the electronic structure of the high temperature superconductors. hore recently, it has been shown ${ }^{1}$ that it is important to cleave $\mathrm{YBa}_{2} \mathrm{Cu}_{3} \mathrm{O}_{7-8}$ ( $Y$ 123) samples at low temperature to ensure that the surface probed by photcemission is characteristic of the bulk. The $\mathrm{Bi}_{2} \mathrm{Sr}_{2} \mathrm{CaCu}_{2} \mathrm{O}_{6}$ (B1 2212) class of superconductors is much more stable at elevated temperature, but reduced temperatures prolong the useful $11 \mathrm{fe}$ of a surface. Additionally, reduced Fermo level broadening is loportant in studying the detalls of band dispersion near the Fermi level. Spectra can be further slopIf jed by angular ( $k$ space) resolution, high kinetic energy resolution, or the application of all three at the same time.

\section{ECPEIYDTIAL}

Beasureaents were done at the Synchrotron Radiation Center" Stoughton, Misconsin on the Anes/Montara state-ERG/Seya bearline ${ }^{2}$. The photoealssion spectroneter has two degree angular resolution and is capable of $20 \mathrm{meV}$ electron resolution. The combined systen resolution in Figs. 3 and 4 was $32 \mathrm{meV}$.

\section{Resucts}

The spectra in Fig. 1 are taken in normal eaission. In this geonetry a shift in the binding energy of a peak as a function of photon energy represents dispersion along the direction normal to the ab plane. As can be seen in Fig. la for B1 2212, there is uinimal dispersion. The priwary effects are a small shift of the $3.5 \mathrm{eV}$ peak to lower binding energy

\footnotetext{
Some support for the presentation of this work relates to Departnent of Navy Grant N00014-89-J-1837 1ssued by the office of Naval Research. The United States Government has a royalty-free ilcense throughout the world in ali copyrightable aterial contained herein.

The Ames Laboratory is operated for USDOE by IOw日 State University under contract No. w-7405-eng-82.

the Synchrotron Radlation Center is supported by NSF under contract $104 R 8601349$.
} 

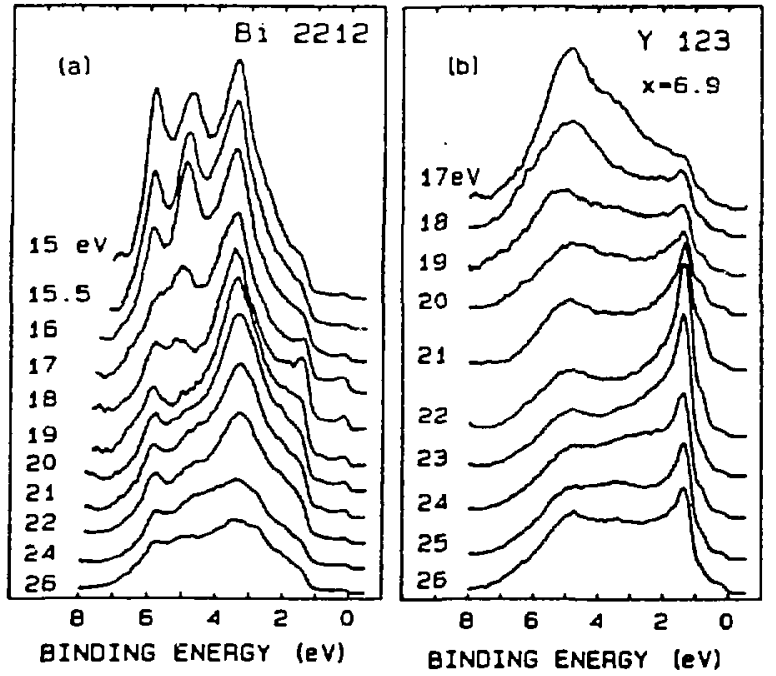

FIGURE 1

Proccemission energy distribution curves taken in normal emission for B1 2212 (a) and $Y 123$ (b). The samples were cleaved and maintained at $20 \mathrm{~K}$. The spectra are normalized to incident photon flux.

with Increasing photon energy and a larger shift of the $4.8 \mathrm{eV}$ peak to higher binding energy. The small amount of dispersion in the 2 direction is not surprising in this highly two dimensional material. Dispersion In less well defined peaks is masked by the very strong intensity modulations due to matrix element effects. The enhanceinent near the Ferml level and at $1.5 \mathrm{eV}$ for photon energies near $18 \mathrm{eV}$ are only one of the strong selection rule effects.

Fig. Ib is a similar set of spectra for Y 123. Again, the two dimensionality of this material results in oinimal dispersion in this geometry. There are indications of dispersion lower in the valence band, but the dominant features are the very strong matrix element effects. From these spectra, and others taken at other emission angles, it is clear that the strong enhancement of the $1.25 \mathrm{eV}$ peak at a 22 eV photon energy 15 a matrix element effect and not an oxygen resonance.
Dispersion is wuch larger along directions in the ab plane. Fig. 2 are spectra for different emission angles and hence different $k$ values along approximatly the $[-5$ direction. An initial state at $1.25 \mathrm{eV}$ splits with one branch remaining at about $3.25 \mathrm{eV}$ and the other moving deeper away froa the zone center. Additional dispersion is seen in the lower part of the valence band. Finally, a band is seen dispersing through the Fermi level. In both Fig. 1 and 2 , because of the coderate resolution (150 nev), emission near the Fermi level is largest when the band is actually well below $E_{f}$. High resolution data taker at the same time as the spectra in Fig. 2 show that the Fermi level crossing is ot 12 degrees.

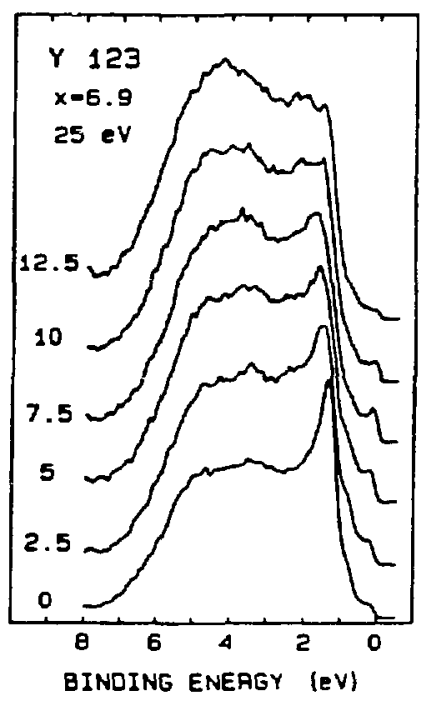

FIGURE 2

Angle resolved photoemission spectra along approximately the $\Gamma-5$ direction, normalized to incident photon flux. The angle is relative to the sample normal.

For B1 2212, emission near the Fermi level can be seen in much greater detall in the high resolution spectra of Fig. 3. As predicted by all band structure calculations, there is 


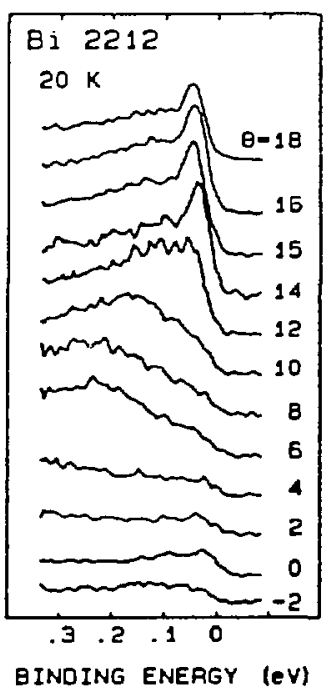

FIGURE 3

High resolution angle resolved spectra along approximately the I-H direction, normalized to incident flux.

minimal emission from the Ferwi level near the zone center. The structure that is seen near normal eraission in the figure probably cones from randonly orfented damaged areas at the edges of the sample. Increasing angles sample a line near the zone diagonal $(I-M)$. $\lambda$ band is seen dispersing towards the Fermi level frod at least 300 meV below $E_{f}$ and then moving away from the Fermi level. This follows the calculated band ${ }^{3}$ qualitativly but the measured band appears much closer to the Ferml level than predicted. This is in qualitative agreement with Takahash1 et.al. 4

The measurements shown in Fig. 3 were taken at 20K, and the shape of the band near the Ferai level is distorted by the existence of a superconducting gap. Data reported in Ref. 5 were taken along a line parallel to that of Fig. 3, and include a point which is one of the few places where a band in the normal state crosses the Fermi level. The spectra ${ }^{5}$ in the normal and superconducting state are shown in the upper part of Fig. 4. Modeling these spectra gave $2 \Delta / \mathrm{KT}_{\mathrm{c}}=7$, twice the weak coupling BCS value. In the bottom half of $F \mathrm{lg} .4$, the sase normal state spectrum is compared to a piatinum foll on the sample holder. The instrumental resolution ( $32 \mathrm{meV}$ ) is comparable to the Ferml level width at this temperature (34 meV). The measured Fermi levels are equivalent to within the resolution and nolse of the experiment. A report by these authors of a more extensive experiment on the normal state Ferol level propertles is in preparation, with the goal of placing quantitative lisits on the possible value of RVB parameters.

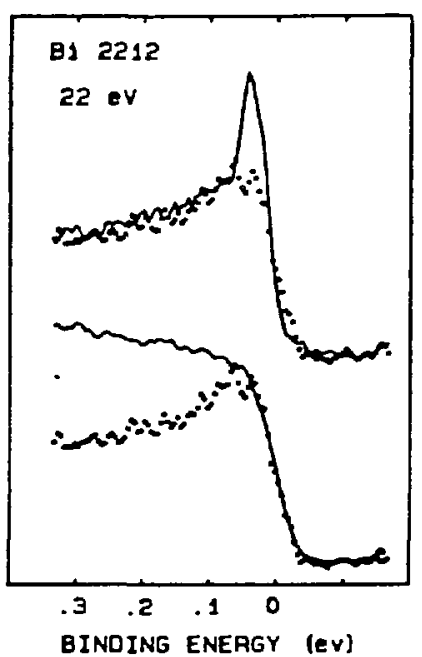

FIGURE 4

Upper: High resolution spectra showing modifications to the density at the Ferml level as a result of a superconducting gap. The solid curve is for 20K. and the dotted curve is for 90K; above the $T$ of $82 K$.

Lower: The same norfhal state density compared to a Pt density of states (solid line) at 90K.

\section{CONCLUSIONS}

High energy and angular resolution combined with low tenperature is giving an increasingly detalled plcture of the high temperature superconductors. Some of the detall, such as 
the importance of matrix element effects, means a complete angle resolved study of the valence bands will be very time consuming. Fortunatly, increased resolution simplifies other parts of the study. We have determined that bands cross the Fermi level at relatively few points in the zone. By 1solating those polnts from the background we have determined the superconducting gap, and determined that cne spectral density in the normal state locks like an ordinary metal. Increasingly, $Y 123$ and $B 12212$ are looking like traditjonal Ferml liquld materlals.
REFERENCES

1. A.J. Arko et. al., J. Mag. Magn. Mat. 75 (1988) Ll.

2. C.G. Olson, Nuc. Inst. Methods in Physics Research 1266 (1988) 205.

3. S. Massidda, J. Yu, and A.J. Freeman, Physica C 52 (1988) 251.

4. T. Takahashl et. al., Phys. Rev. B39 (1989) 6636.

5. C.G. Olson et.al., Science (1989) in press. 